Volume 9, No.4, July - August 2020

International Journal of Advanced Trends in Computer Science and Engineering

Available Online at http://www.warse.org/IJATCSE/static/pdf/file/ijatcse221942020.pdf

https://doi.org/10.30534/ijatcse/2020/221942020

\title{
Performance Anatomization of Routing Protocols in Wireless Sensor Network
}

\author{
Mohamed Najmus Saqhib ${ }^{1}$, Lakshmikanth.$S^{2}$ \\ ${ }^{1}$ Visvesvaraya Technological University, Research center VKIT Bangalore, India, Saqhibkhan89@gmail.com \\ ${ }^{2}$ Acharya Institute of Technology, India, Lakshmikanth18@gmail.com
}

\begin{abstract}
Wireless Sensor Network (WSN) is used in multifarious applications like environment monitor, battle based systems, enemy vehicle track determination and many more. It is also limited by various constraints like cost, bandwidth, and energy consumption patterns along with network lifetime. When the data packets have to be sent to the destination node or control center after detection, the path is established between the detected node and the destination node [1]. When the number of paths is more and nodes repeatedly participate in those paths then residual energy value is also reduced of the specific nodes which lead to holes in the network and reduces the network lifetime. This paper presents an overview of WSN, Lifetime ratio effects, a numerical survey of the energy-efficient routing protocol. The methods namely Destination Sequence Distance Vector (DSDV), Ad hoc On-Demand Distance Vector (AODV), Zone Routing Protocol (ZRP) and Energy Efficient Distance Routing (EEDR) are discussed in detail along with the implementation of these methods in MATLAB. Comparison is performed in terms of various parameters namely delay, hops, energy consumption, alive nodes, dead nodes, lifetime ratio, overhead ratio, residual energy as well as throughput [10] and it is proved that EEDR algorithm works in an optimized fashion.
\end{abstract}

Key words: AODV, DSDV, EEDR, Energy Efficiency, Lifetime Ratio, Wireless Sensor Network.

\section{INTRODUCTION}

Wireless Sensor Network (WSN) is a micro service based system which can be used for applications varying from commercial and industrial data. The features of WSN includes a processor, communication protocols, amount of power used and the path between the two nodes or between the base station and the node [2]. Each node can perform various tasks ranging from detecting temperature, humidity data and pressure data based transmissions between two endpoints. The WSN can be used in a wide variety of services namely Military, Industry, Health Care and many more [6].
WSN consists of a set of nodes which are spread in a given area of $x^{*} y$ meters. Each node is defined with a unique value of (xi, yi). xi is the ith position for the node, yi is the y position of the node in a two-dimensional space[9]. Each of the nodes is also identified with a unique id representing itself. Figure 1 shows the node placement strategy for the set of 100 nodes spread across $100 * 100$ area. The node ids are varied from Node1 to Node100.

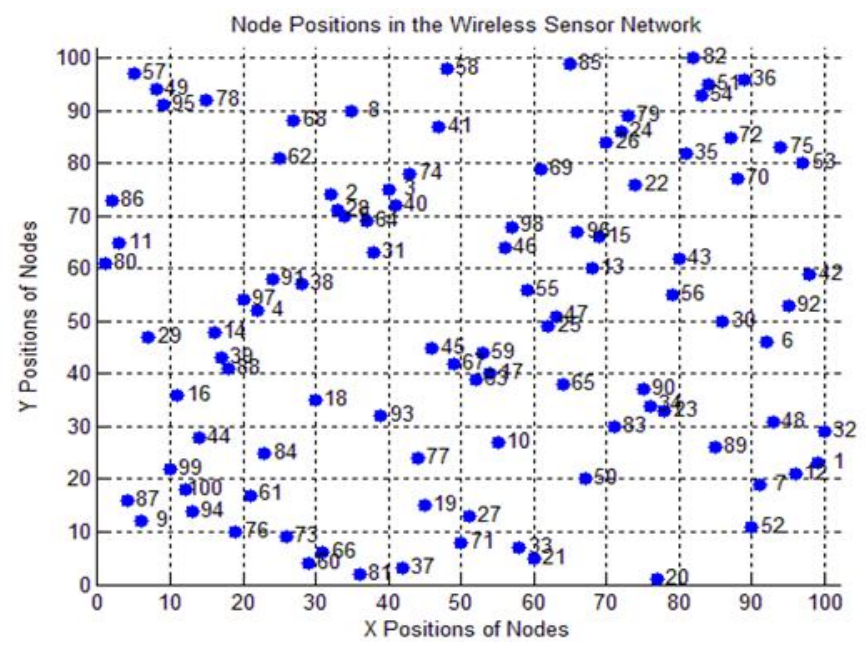

Figure 1: Node Placement Strate gy

As shown in Figure.1, the following are the positions of a few of the nodes among the 100 nodes in the network.

Table 1: Node position in the network

\begin{tabular}{|c|c|c|}
\hline Node ID & $\begin{array}{c}\text { X Position of } \\
\text { the Node }\end{array}$ & $\begin{array}{c}\text { Y Position of the } \\
\text { Node }\end{array}$ \\
\hline 11 & 9 & 67 \\
\hline 9 & 7 & 11 \\
\hline 37 & 42 & 4 \\
\hline 31 & 39 & 65 \\
\hline 84 & 22 & 27 \\
\hline
\end{tabular}

One of the major challenges is maintaining the [4], [5] lifetime ratio for the network. If IE is the initial energy of the network then a node which has the threshold satisfying the equation as below is calculated.

$R E \geq I E / 4$

(1) 
And the set of nodes who do not satisfy the equation are taken in the denominator to obtain the lifetime ratio.

$L R=$ no of $R E \geq \frac{Z E}{4}$ to no of $R E<I E / 4$

If there are no nodes which are having the remaining energy (RE) below IE/4 then LR will be moving towards infinity and vice versa. When the nodes take part in the data portage their consumed energy depends on the various factors like attenuation factor (att), the energy required for amplification (Eamp), the energy required for data packet transmission (Etxn) along with the distance between the nodes in the network [8].

Econsumed $=2 *$ Etxn + Eamp $*$ distance ${ }^{\text {at }}$

Each time a node will be used in the path the energy level for the nodes will be reduced by [20]

$R E=C E-$ Econsumed

Where CE is the current energy of the node during the computation of RE. If the value of energy required for transmission, amplification and attenuation factor is kept constant then energy variation can be plotted as in Figure 2 .

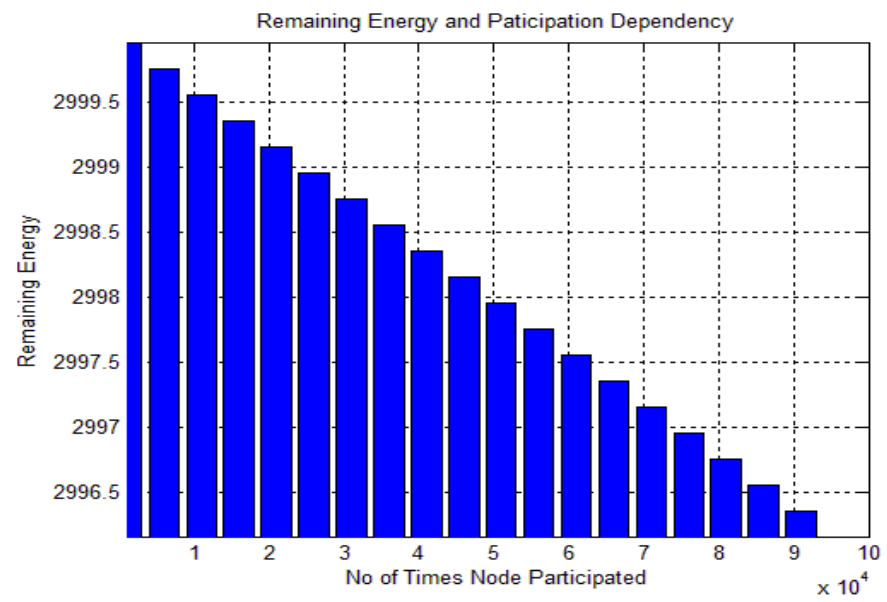

Figure 2: Energy distance dependence

Figure 3 shows residual energy reduction for a specific node whose initial energy level is $3000 \mathrm{~J}$ is reduced with the number of time the node participates. For the $1^{\text {st }}$ time, it is $3000 \mathrm{~J}$ and at the end of 10 iterations, the residual energy is $2995 \mathrm{~J}$.

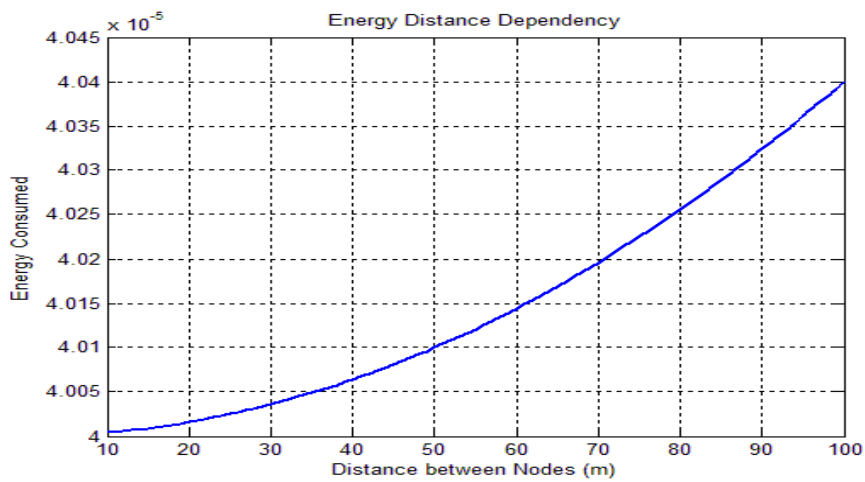

Figure 3: Residual Energy Reduction with the participation ratio The reduction in energy happens using the following logic
$U E=C E-E C$

Where UE is updated energy level [7], CE is the current energy level and $\mathrm{Ec}$ is the energy consumed. For instance, if the energy required is assumed as 20J, energy for amplification is $10 \mathrm{~J}$, the distance between two nodes is 10.34 $\mathrm{m}$ and environment factor is 0.7 with the node initial energy value as $3000 \mathrm{~J}$ then the updated energy level can be computed as below

$U E=3000-\left(2 * 20+10 * 10.34^{\wedge} 0.7\right)=2908.703 \mathrm{~J}$

The remaining part of the paper is organized as follows first the existing work present in the literature is presented, secondly, the EEDR method is discussed in detail, thirdly several existing methods namely DSDV, AODV and ZRP are discussed. The final section determines the results of the various algorithms and comparison between the algorithms

\section{ROUTING PROTOCOLS}

The several existing methods [10] which are present in the literature are described in this section namely AODV, DSDV and ZONE based routing method.

\subsection{DSDV routing protocol}

The table used in the DSDV method [16], will maintain the path information along with node information with respect to unique destinations. For every period of Time 'T,' the routing information gets updated. First, from the detection point, the nodes which are occurring inside the transmission scope are found out and then the path is determined from each detection point to control center.

The time taken is found for each of the paths and the path which has the lowest time taken is chosen for packet delivery. Consider a set of nodes which are arranged in the format of a line in which each node is separated at a distance of $10 \mathrm{~m}$.

$$
1--\rightarrow 2-\rightarrow 3-\rightarrow 4-\rightarrow 5-\rightarrow 6-\rightarrow 7-\rightarrow 8
$$

If the initiator is Node1 and the transmission range is $20 \mathrm{~m}$ then the set of nodes which form the cover set of Node1 is \{Node2, Node 3$\}$ and if the initiator is Node 3 then set of cover set nodes are $\{$ Node1, Node2, Node4 and Node5\}. The initiators in the DSDV method are found out using the algorithm described in Figure 4.

The multiple paths from the initiator nodes to the destination node are found out using the following algorithm as described in Figure 5.

The individual path is found out using the algorithm described in Figure 6. 
Input- SN and Transmission Range Description

7. $\mathrm{SN}, \mathrm{DN}$, Transmission range

8. Find the nodes in transmission range with respect to $\mathrm{SN}$ $\mathrm{N}=\{\mathrm{n} 1, \mathrm{n} 2, \ldots \ldots, \mathrm{ncs}\}$ where ni represents the ith node

9. Measure the length of $\mathrm{N}$

10. Initialize the initiator set $\{$ IS $\}$

11. For $\mathrm{i}=1 ; \mathrm{i}<=\mathrm{N} ; \mathrm{i}++$

d. Pick the ith node ni

e. Find the nodes which are within the transmission range of ni call it as TRi

f. Store the Tri into $\{$ IS $\}$

12. The final set will contain

$\{\mathrm{TR} 1, \mathrm{TR} 2, \ldots \ldots . ., \mathrm{TRn}\}$

Figure 4: Initiator Nodes Determination DSDV

\author{
Determination \\ Input - Initiator Nodes, DN, Transmission Range \\ Algorithm \\ 1. Find the number of initiator nodes $(\mathrm{Nl})$ \\ 2. For $\mathrm{i}=1, \mathrm{i}<=\mathrm{Nl} ; \mathrm{i}++$ \\ a. Start the Timer \\ b. Execute the IPM method to find the path with ith node \\ acting as $\mathrm{SN}$ \\ c. Stop the Timer \\ d. Find the time difference \\ e. Store the Map $\{\mathrm{Ti}$, Pathi, $\}$ \\ f. Store the time Ti in List \\ Output - List and Map \\ List has all the time taken across the routes \\ Map has the path and the respective time taken
}

Figure 5: Multi-Path Determination

\author{
Individual Path Method (IPM) \\ Input \\ SN, DN and Transmission Range \\ Description \\ 1. SN, DN and Transmission Range \\ 2. Find the nodes which belong to transmission range acting as \\ Cover Set (CS) \\ 3. If the DN belongs to CS, then Stop \\ 4. If DN is not present in CS then find the subset of CS as \\ Forward Nodes (FN) \\ 5. The rules are generated from the $\mathrm{FN}$ \\ 6. The node will be picked based on rule \\ 7. Process is repeated until path is completed
}

Output: Path between SN and DN

Figure 6: Individual Path Determination DSDV

The multiple paths are found and then the cache of route discovery time is maintained in the format of \{tDSDV1, tDSDV2... tDSDVn $\}$. Where tDSDV1 is the time taken to find the path1 using DSDV algorithm, tDSDVn is the time taken to find the path $\mathrm{n}$ using DSDV algorithm. The best path is found out by making use of a minimization principle used by the following formula

btDSDV $=m i n\{t D S D V 1, t D S D V 2, \ldots t D S D V n\}$

The path which corresponds to $t D S D V n$ will be used to deliver the data packets.

\subsection{AODV routing protocol}

In AODV the path maintenance overhead is reduced since every node does not maintain the cache of route [13]. From all the nodes in the network, a set of nodes are chosen for storage of route and route initiation is done only if it is required [17]. The AODV is built by modifying the DSDV algorithm. The number of initiator nodes in the AODV is less compared to DSDV algorithm. After all the paths are found out the path which has the lowest end to end distance is chosen as the best path.

The initiator nodes are found out by making use of the following algorithm as described in Figure 7.

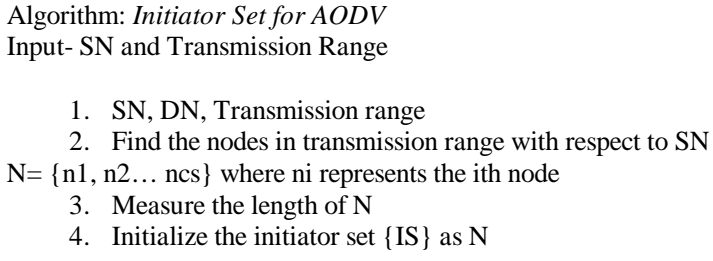

Figure 7: Initiator Nodes Formation AODV.

The multiple-path determination for the AODV algorithm can be determined by making use of the Multipath Routing AODV method as described in figure 8.

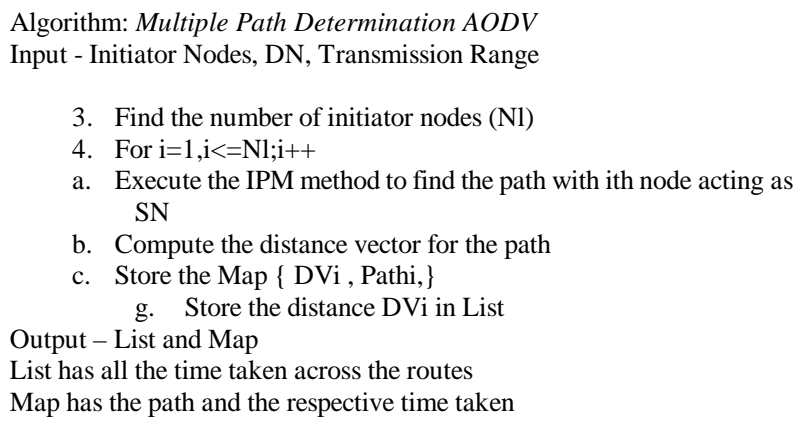

Figure 8: Multiple Path Determination AODV.

The objective function for determining the path sequence for data transmission is defined as below

bDistance $=\min (D V p 1, D V p 2, \ldots, D V p n)$

Where DVpi is the distance on the path pi. After finding the minimum distance vector then the best path is found out 


\subsection{Zone Routing Protocol (ZRP)}

The border nodes are found out from the neighbor nodes [18]. The path is found out from the border nodes using individual zone routing path. From the set of multiple routes route with best time value is found out. The individual path zone routing is found out as described in Figure 9

\section{Algorithm: Individual Path Method (IPM) ZRP}

Input - SN, DN and Transmission Range

1. SN, DN and Transmission Range

2. Find the nodes which belong to transmission range acting as Cover Set (CS)

3. If the DN belongs to CS, then Stop

4. If DN is not present in CS then find the round trip time with respect to CS nodes

5. The node will be picked based on lowest round trip time

6. Process is repeated until path is completed

Output -Path between SN and DN using ZRP

Figure 9: Zone Routing Protocol Path Discovery Routine

\subsection{Energy-Efficient Distributed Receiver (EEDR) routing protocol}

The receiver node will find the CRN packets to all nodes. For each of the nodes in the cover set quality of the channel is found out.[3] The maximum value of channel quality \{CQI1, CQI2, CQIn \} is found out and then the next node is found out based on the maximum value. The initiator nodes are found out based on AODV method. The CQI based selection happens until TTL becomes zero. Once the TTL becomes 0 then the shortest path method is triggered. The individual path is found out by using the algorithm as described in Figure 10.

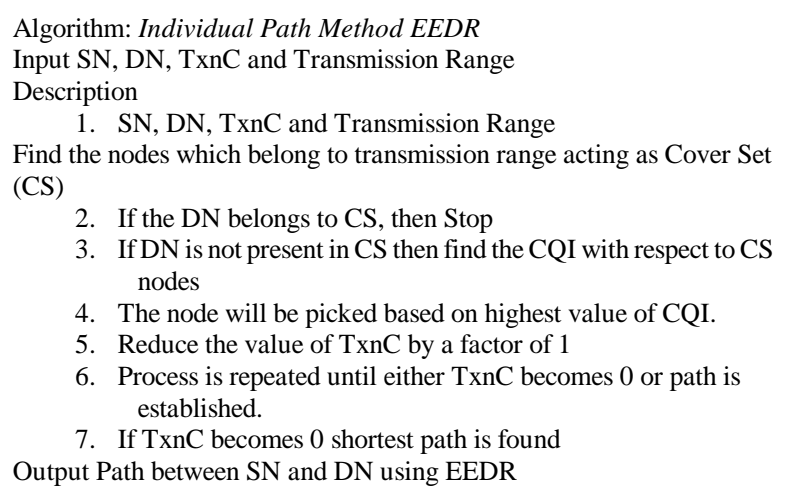

Figure 10: EEDR Individual Method

The best route is found out by making use total CQI value which is having a maximum value. The CQI will be computed by making use of the signal to noise interference ratio (SNIR) on the block-based transmission packets send from the source node. The rate of transmission and the kind of modulation technique is used to find the channel state information. The Channel Quality Indicator (CQI) is found using the following formula

$C Q I=\left|\frac{S N I R}{1.02}+16.62\right|$

$-16<$ SNIR $<14$

Where

$\mathrm{CQI}=$ Channel Quality Indicator

SNIR = Signal to Noise Interference Ratio

The SNIR can be defined as follows

$S N I R=\frac{\frac{P_{T}}{L_{P}}}{N_{Q} W F} P G$

Where

$$
\begin{aligned}
& \quad \mathrm{N}_{Q}=1.38: 10^{-23} * 2 \\
& \mathrm{P}_{\mathrm{T}}=\text { Transmitted Power } \\
& \mathrm{L}_{\mathrm{P}}=\text { Path Loss } \\
& \mathrm{W}=\text { Signal Bandwidth } \\
& \mathrm{F}=\text { Noise Figure } \\
& \mathrm{PG}=\text { Power Gain }
\end{aligned}
$$

The shortest path algorithm will be described in Figure 11

\author{
Algorithm: Shortest Path Method \\ Input \\ SN, DN and Transmission Range \\ Description \\ 1. SN, DN, TxnC and Transmission Range \\ 2. Find the nodes which belong to transmission \\ range acting as Cover Set (CS) \\ 3. If the DN belongs to CS, then Stop \\ 4. If DN is not present in CS then find the distance \\ with respect to CS nodes \\ 5. The node will be picked based on lowest value of \\ distance. \\ 6. Repeat process until DN is reached. \\ Output Path between SN and DN using Shortest Path
}

\section{RESULT}

Figure 11: Shortest Path Method

This section will present the comparison results of EEDR, DSDV, AODV and ZRP algorithms. The simulation input is defined in Table 2. The results are based on the MATLAB programming language. 
Table 2: Node position in the network

\begin{tabular}{|l|l|l|}
\hline Parameter Name & Parameter Value \\
\hline Number of Nodes & 100 \\
\hline Source Node & 71 \\
\hline Destination Node & 8 \\
\hline Transmission Range & 40 \\
\hline Initial Energy For Nodes & $9999 \mathrm{~mJ}$ \\
\hline $\begin{array}{l}\text { The energy required for } \\
\text { transmission }\end{array}$ & $20 \mathrm{~mJ}$ \\
\hline The energy required for & $10 \mathrm{~mJ}$ & \\
amplification & & \\
\hline Attenuation Factor & 0.5 & \\
\hline Threshold Count & 4 & \\
\hline Number of Iterations & 25 & \\
\hline
\end{tabular}

Figure 12: Node Formulation

Figure 12 shows the Node Formulation Module Output. As shown in the figure the nodes are spread in a $100 * 100$ area. Each Node is assigned a unique ID and there are 100 nodes in the network. Node 71 is placed at the location $(1,10)$, Node 3 is present at the location $(80,22)$.

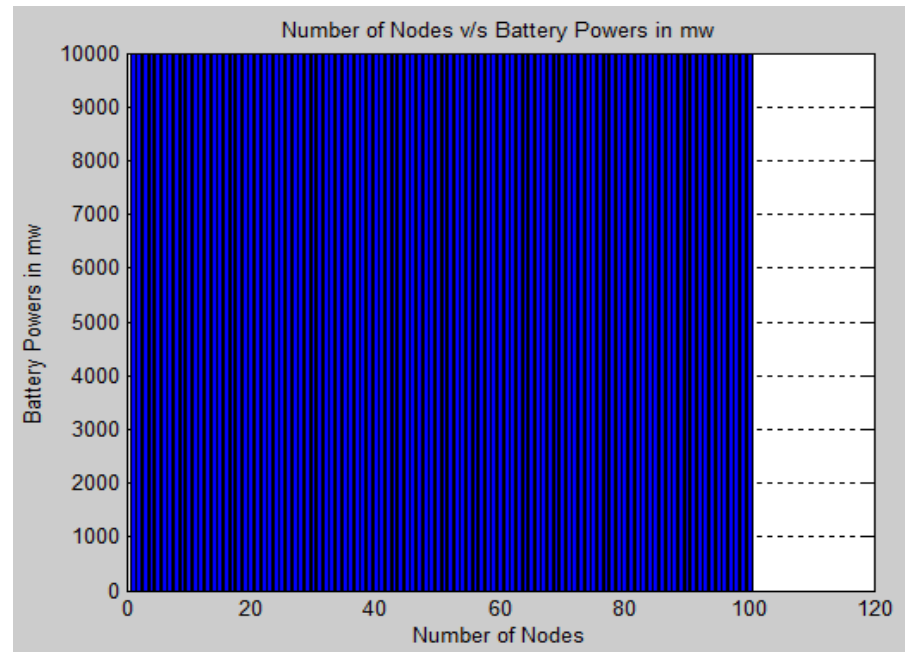

Figure 13: Initial Battery Level for the Nodes
Figure 13 shows the initial battery level for all the nodes in the network. All the 100 nodes have been initialized with a value of $9999 \mathrm{~mJ}$.

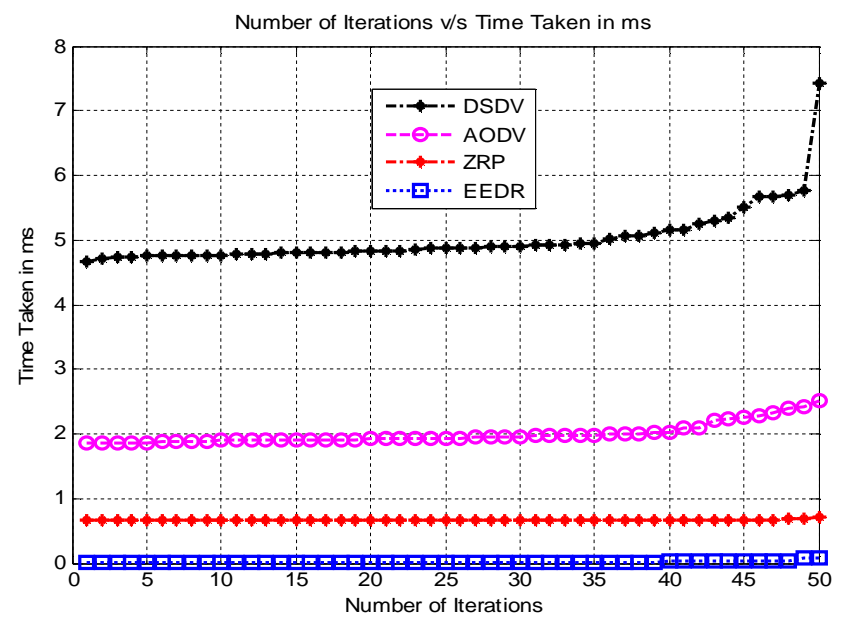

Figure 14: Delay Comparison

Figure 14 shows the delay comparison of the various methods. The $\mathrm{X}$-axis the representation of the number of times all the algorithms are executed and Y-axis is the time taken for the entire path. EEDR has the lowest delay as compared to other methods namely DSDV, AODV and ZRP. The EEDR algorithm has a delay range between 0.01 to $0.02 \mathrm{~ms}$. ZRP has a delay in the range of 0.08 to $0.09 \mathrm{~ms}$. AODV has a delay in the range of 1.8 to $2.5 \mathrm{~ms}$. DSDV has a delay in the range of 4.3 to $7.5 \mathrm{~ms}$.
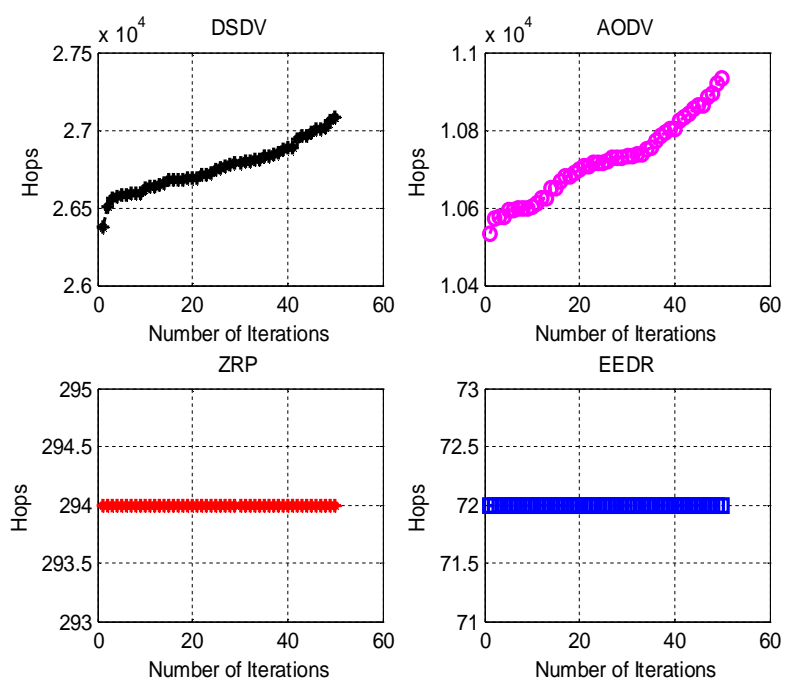

Figure 15: Hops Comparison

Figure.15 shows the hops comparison for the period of 50 iterations. As shown in Fig the DSDV algorithm has the hops in the range of 26500 to 27200 hops which is for all the possible paths in the network. AODV will have the hops in the range of 10600 to 10900 hops. ZRP has the hops of around 294 while EEDR has the lowest hops of 72 across all paths in 
the network. The performance of EEDR is optimum for hops as compared to other methods as shown in the graph
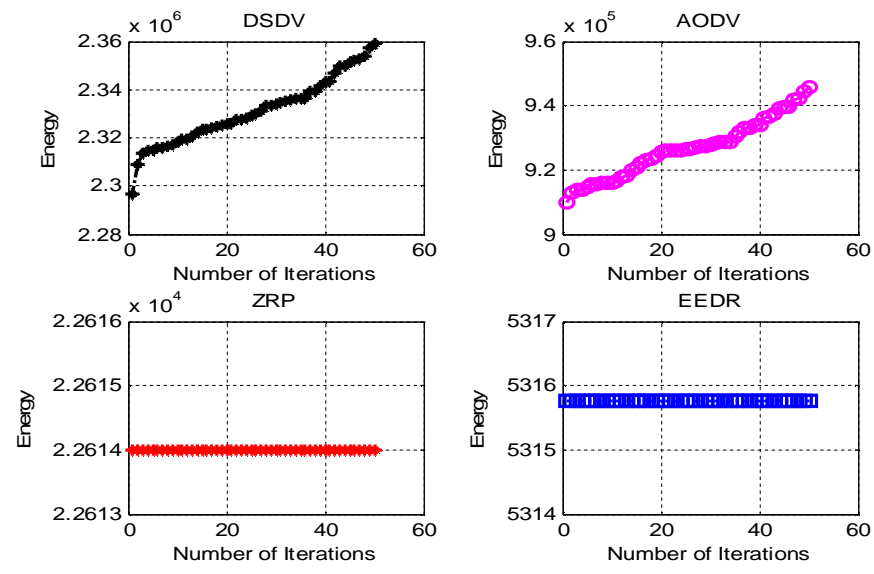

Figure 16: Energy Consumption Comparison

Figure.16 shows the energy consumption comparison of the various methods... As seen in the figure the highest energy consumption exists for DSDV method in the range of $2.29 \mathrm{MJ}$ to $2.36 \mathrm{MJ}$. AODV method has the next highest energy consumption in the range of $0.9 \mathrm{MJ}$ to $0.95 \mathrm{MJ}$. ZRP has the highest energy consumption in the range of $22.6 \mathrm{KJ}$ to 22.7 KJ. EEDR has the least energy consumption in the range 5315.2 $\mathrm{J}$ to 5315.8J. EEDR has the lowest energy consumption followed by ZRP, AODV and DSDV.

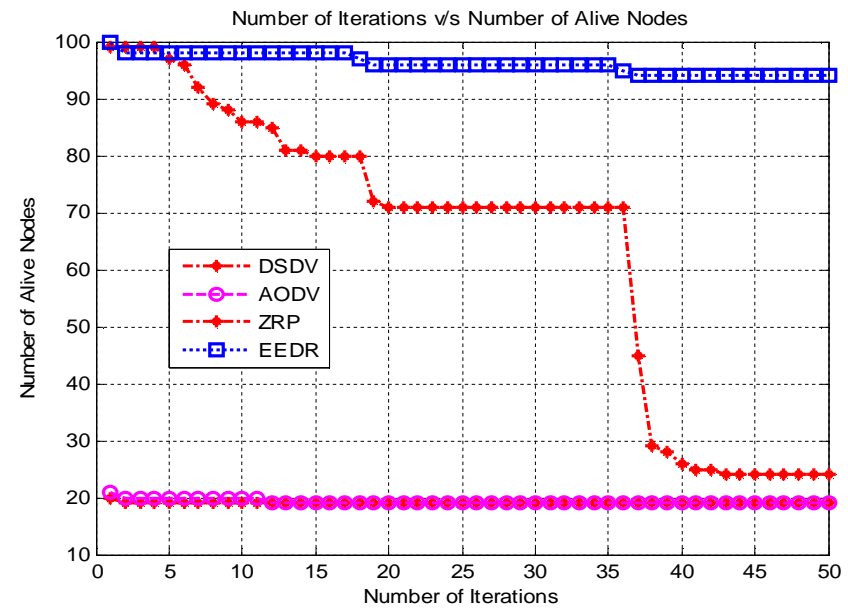

Figure 17: Number of Alive Nodes

Figure. 17 shows a comparison of the number of alive nodes. The nodes whose value is above or equal to the threshold value of (9999/4) is defined as the $y$-axis. The alive nodes count at the end of 50 iterations for EEDR algorithm is defined as 94, followed by ZRP which has 22 alive nodes, AODV has the next number of alive nodes with a value of 19 nodes along with DSDV.
Figure.18 shows a comparison of the number of dead nodes. The nodes whose value is less than the threshold value of (9999/4) is defined as the Y-axis. The dead nodes count at the end of 50 iterations for EEDR algorithm is 8 nodes, followed by ZRP which has 78 dead nodes, AODV has the next number of dead nodes with a value of 81dead nodes along with DSDV

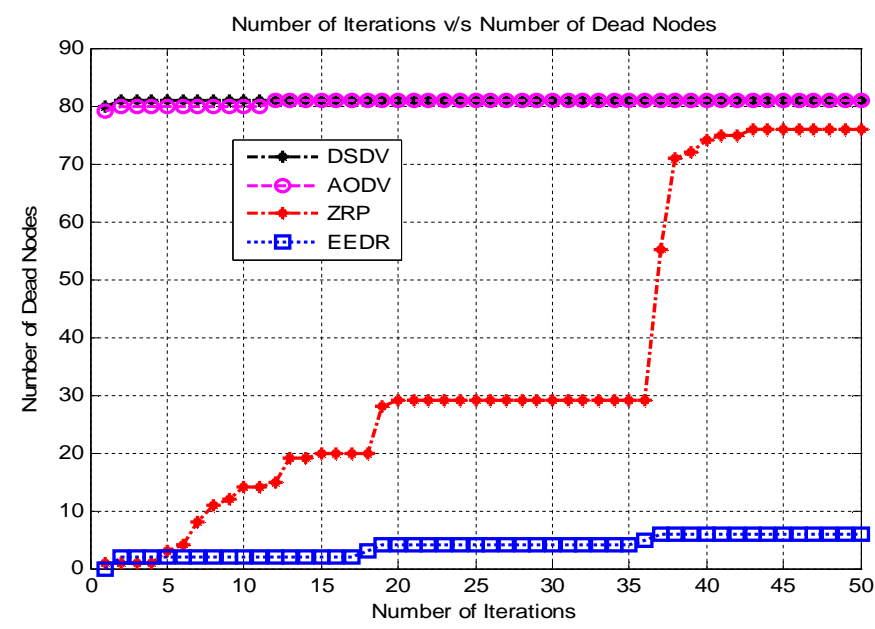

Figure 18: Number of Dead Nodes

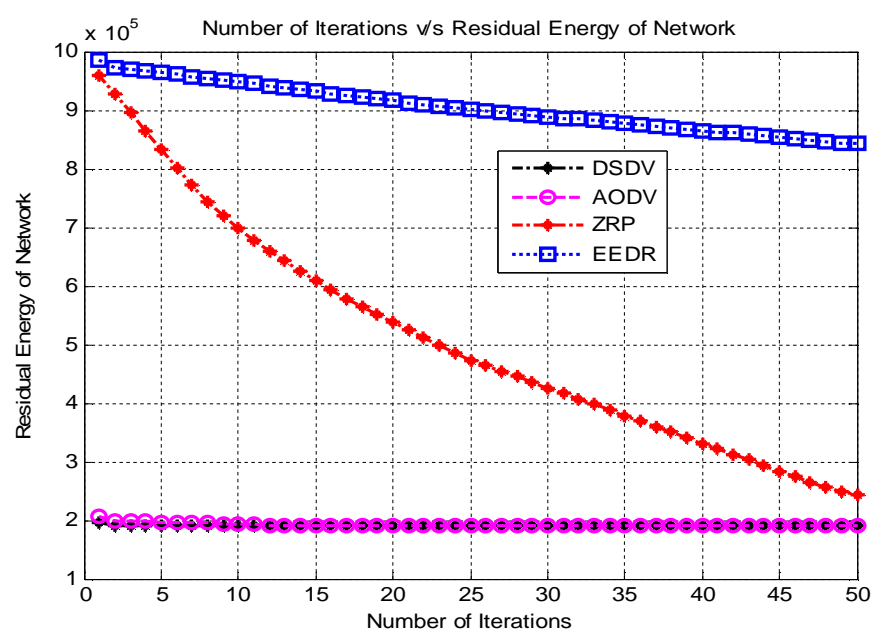

Figure 19: Residual Energy for Nodes

Figure.19 shows the residual energy for nodes. As shown in the Figure EEDR algorithm has the highest residual energy followed by ZRP, AODV and DSDV. At the end of 50 iterations, $88 \mathrm{~kJ}$ of remaining energy exists in the network for EEDR algorithm, followed by ZRP which has the residual energy value of $25 \mathrm{~kJ}$. AODV and DSDV have residual energy of around $2 \mathrm{~kJ}$. Also, observe the graph when the number of iterations increases the residual energy of the network decreases.

Figure 20 shows the comparison of packets dropped across the various algorithms namely EEDR, ZRP, AODV and DSDV. At the end of 50 iterations, there is maximum packet drop of 10 packets for EEDR followed by ZRP with a maximum packet drop of 20, For AODV the number of packets dropped is 25 and for DSDV it is 35 


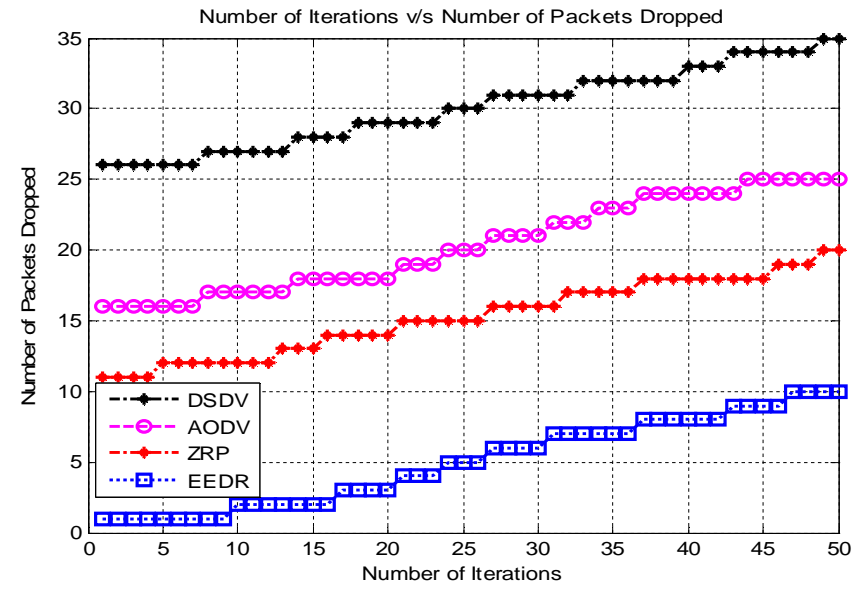

Figure 20: Number of Packet Drop

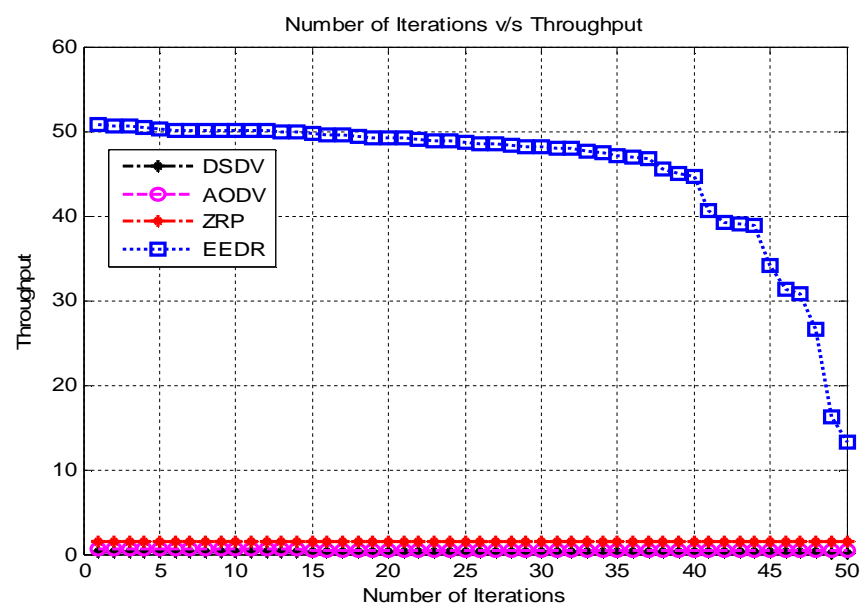

Figure 21: Throughput Comparison

Figure 21 shows the throughput comparison between EEDR, ZRP, AODV and DSDV algorithms. As shown in the fig EEDR has the highest value of throughput followed by ZRP, AODV and DSDV method. Also as the number of iterations increases the value of throughput reduces across all the algorithms

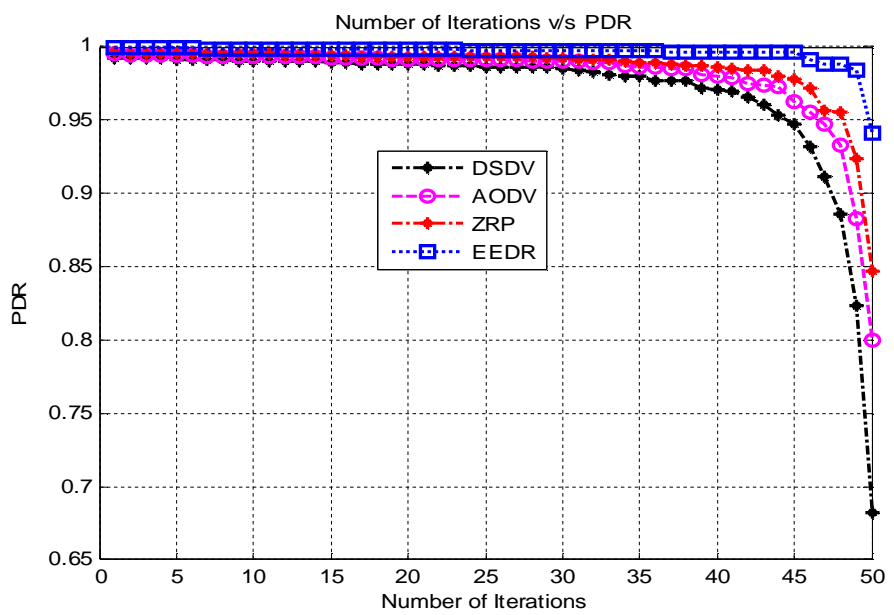

Figure 22: Packet Delivery Ratio
Figure 22 shows the packet delivery ratio comparison for all the 4 algorithms for a period of 50 iterations. At the end of 50 iterations, the EEDR packet delivery ratio remains up to $94 \%$. For ZRP it is $85 \%$, AODV $80 \%$ and DSDV being the worst at $63 \%$.
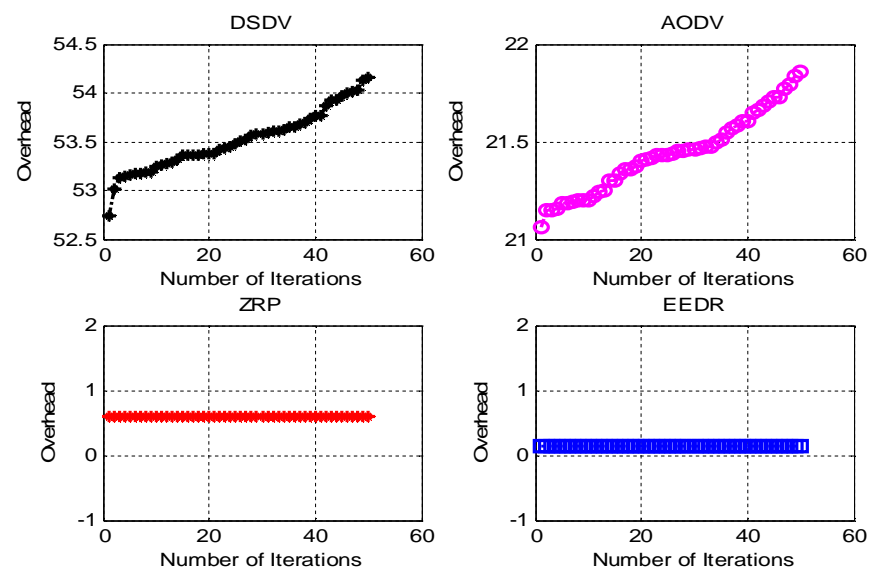

Figure 23: Overhead Measure

Figure 23 shows the overhead comparison between the algorithms. DSDV has the highest value of 54.2 as the overhead followed by AODV being 21. ZRP has an overhead of 0.8 and least is 0.2 for EEDR algorithm.

\section{DISCUSSION}

The percentage-wise measure of parameters across the spectrum of algorithms - EEDR, ZRP, AODV and DSDV.

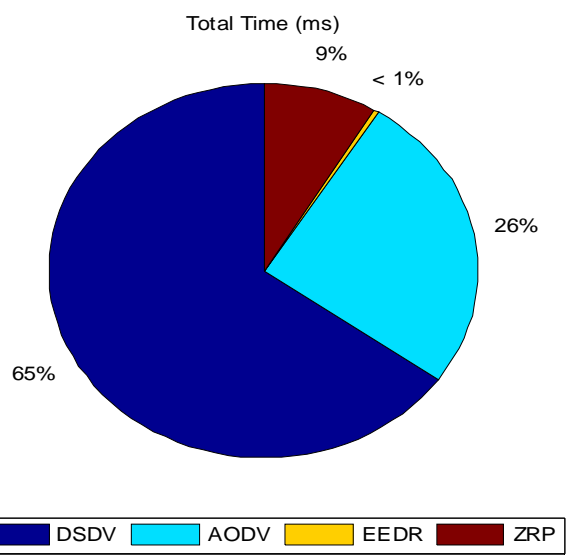

Figure 24: Delay Comparison Percentage

Figure 24 shows the delay comparison percentage the DSDV algorithm has $65 \%$ utilization of time hence will have very less time performance, followed by AODV which occupies around $26 \%$ of the total execution time. $9 \%$ is time utilized by ZRP and least is EEDR which occupies the time less than $1 \%$. 


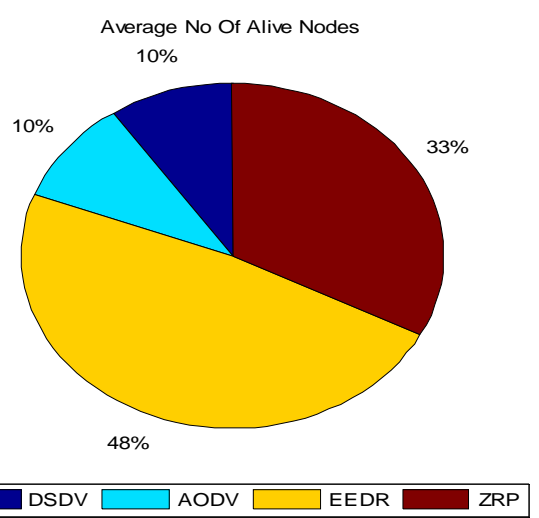

Figure 25: Alive Nodes Comparison Percentage

Figure. 25 shows the alive nodes comparison. $48 \%$ percentage of the alive nodes are for EEDR algorithm, followed by ZRP $33 \%, 10 \%$ each for AODV and DSDV methods

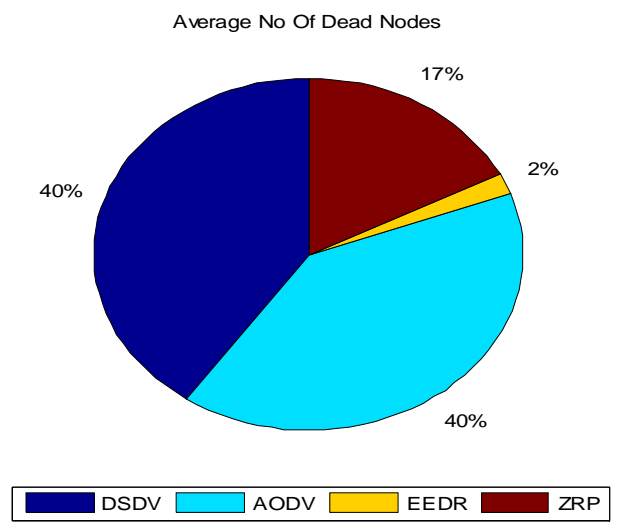

Figure 26: Dead Nodes Comparison Percentage

Figure 26 shows the dead node comparison percentage. EEDR has the least dead node percentage of $2 \%$ followed by ZRP which is $17 \%$, AODV and DSDV have an equal distribution of $40 \%$ dead nodes

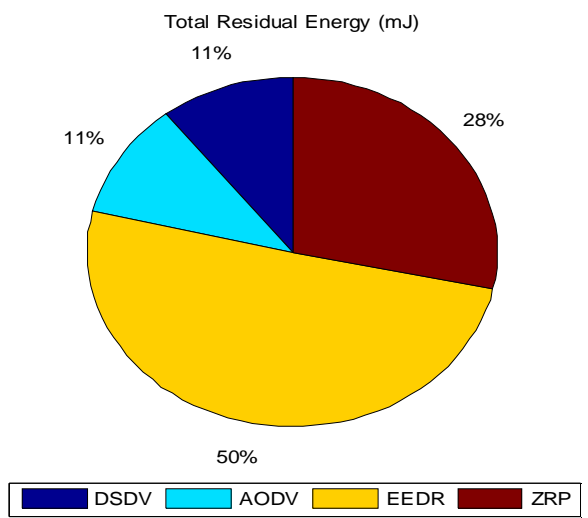

Figure 27: Total Residual Energy
Figure. 27 shows the residual energy comparison. $50 \%$ of the total network residual energy is occupied by EEDR, followed by ZRP with a value of $26 \%$, AODV and DSDV have a value of $11 \%$.

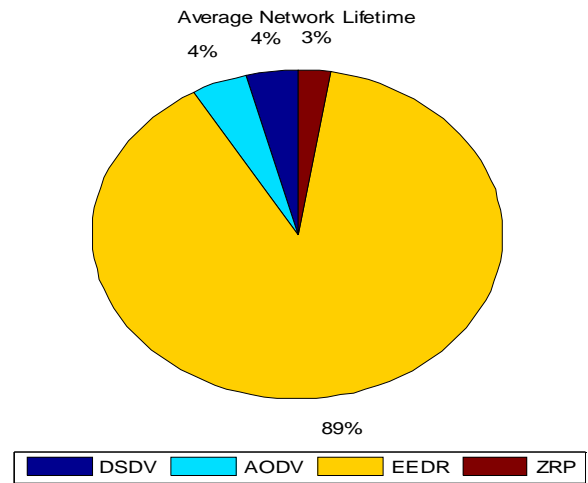

Figure 28: Average Network Lifetime

Figure 28 shows the average network lifetime. EEDR has occupied around $89 \%$ of average network lifetime and remaining three algorithms have $4 \%$ of overall average network lifetime.
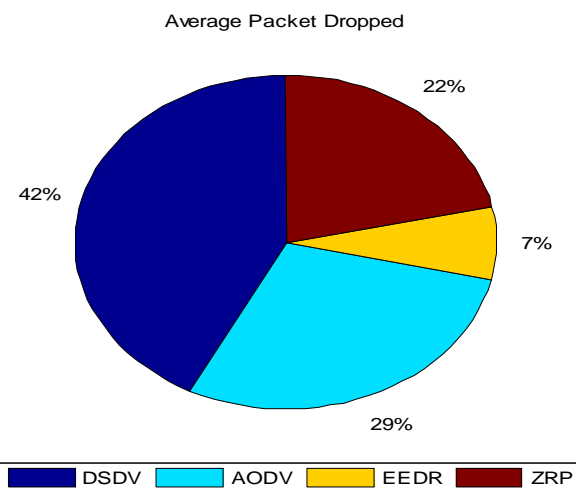

Figure 29: Average Packet Drop

Figure 29 shows the average packet drop EEDR has 7\% average packet drop, ZRP has average packet drop of $22 \%$, AODV has the average packet drop of $29 \%$ and DSDV has the average packet drop of $42 \%$.

\section{CONCLUSION}

The routing methods namely DSDV, AODV, ZRP and EEDR methods are described. The energy consumption analysis along with network lifetime measure is performed. The Simulation results for EEDR, DSDV, AODV and ZRP concerning various parameters namely delay, hops, energy consumed, alive nodes, dead nodes, lifetime ratio, routing overhead, residual energy and throughput are compared. 
The average network lifetime in EEDR is comparatively higher, nearing $89 \%$, than other routing methods. The delay in path establishment is less in EEDR as well as ZRP with the former performing better. The EEDR also saves big on energy whereas DSDV scores are very poor. Thus we see that for all the parameters considered, EEDR behaves in the best fashion. Further study should be carried to study the performance of these algorithms under different buffer policies.

\section{REFERENCES}

1. Kun Xie, Lele Wang, Xin Wang, Gaogang Xie, Jigang Wen, "Low Cost and High Accuracy Data Gathering in WSNs with Matrix Completion", IEEE Transactions on Mobile Computing, Vol 17, PP. 1595 - 1608. 2018.

2. J. Matamoros and C. Antòn-Haro, Opportunistic power allocation and sensor selection schemes for wireless sensor networks, IEEE Transactions on Wireless Communications, vol. 9, pp. 534-539, February 2010. https://doi.org/10.1109/TWC.2010.02.090215

3. Pramod Mutthigarahalli Shankarappa Shankar Performance analysis of EEDR routing protocol for WSNs, IET Wireless Sensor Systems, Vol 7, pp. $21-26$. February 2017,

4. J. Kim, X. Lin, N. B. Shroff, and P. Sinha, Minimizing delay and maximizing lifetime for wireless sensor networks with anycast, IEEE/ACM Transactions on Networking, vol. 18, pp. 515-528, April 2010.

5. L. Van Hoesel, T. Nieberg, J. Wu, and P. J. M. Havinga, Prolonging the lifetime of wireless sensor networks by cross-layer interaction, IEEE Wireless Communications, vol. 11, pp. 78-86, December 2004. https://doi.org/10.1109/MWC.2004.1368900

6. R. Madan, S. Cui, S. Lall, and A. Goldsmith, Modeling and optimization of transmission schemes in energy-constrained wireless sensor networks, IEEE/ACM Transactions on Networking, vol. 15, pp.1359-1372. December 2007.

7. D. Baumann, F. Mager, M. Zimmerling and S. Trimpe, Control Guided Communication: Efficient Resource Arbitration and Allocation in Multi-Hop Wireless Control Systems, IEEE Control Systems Letters, vol. 4, pp. 127-132. Jan. 2020.

8. Q. Zhao and M. Gurusamy, Lifetime maximization for connected target coverage in wireless sensor networks, IEEE/ACM Transactions on Networking, vol. 16, pp. 1378-1391. December 2008.

9. Tajudeen O. Olasupo, Carlos E. Otero, Framework for Optimizing Deployment of Wireless Sensor Networks, IEEE Transactions on Network and Service Management, Vol 5, pp. 1105-1118, September 2018.

10. S. M. Adam and R. Hassan, Delay aware Reactive Routing Protocols for QoS in MANETs: a Review, Journal of Applied Research and Technology, vol. 11, pp. 844-850, 2013.

https://doi.org/10.1016/S1665-6423(13)71590-6
11. J. Matamoros and C. Antòn-Haro, Opportunistic power allocation and sensor selection schemes for wireless sensor networks, IEEE Transactions on Wireless Communications, vol. 9, pp. 534-539, February 2010.

12. Jamal N. Al-Karaki and Ahmed E. Kamal, Routing Techniques in Wireless sensor networks: a survey, IEEE Wireless Communications, pp. 6-28, 2004

13. Saloua Outazgui, Youssef Fakhri RE-AODV an Enhancement of AODV Routing Protocol for Wireless Sensor Networks, in Proc International Journal of Advanced Trends in Computer Science and Engineering, pp. 3565-3569, December 2019 https://doi.org/10.30534/ijatcse/2019/138862019

14. A. Norouzi, M. Dabbaghian, A. Hatamizadeh and B. Berk Ustundag, An Improved EL Gossiping Data Distribution Technique with Emphasis on Reliability and Resource Constraints in Wireless Sensor Network, in Proc IEEE International IEEE Conference on Electronic Computer Technology, Kuala Lumpur, 2010 pp. 179-183.

15. E. Fu, H. Gao, M. Fasehullah and L. Tan, A gradient tracking method for resource allocation base on distributed convex optimization, 2019, in Proc 3rd International Symposium on Autonomous Systems (ISAS), Shanghai, 2019, pp. 41-46.

16. Mahdipour, E., Rahmani, A.M, Aminian, E. Performance Evaluation of Destination-Sequenced Distance-Vector (DSDV) Routing Protocol, in Proc International Conference on Future Networks, Bangkok, 2009.

17. Chakeres, I.D., Belding-Royer, E.M., AODV routing protocol implementation design, in Proc 24th International Conference Distributed Computing Systems Workshops, Tokyo, 2004. https://doi.org/10.1109/ICDCSW.2004.1284108

18. Z. Shafiq, S. A. Mahmud; G. M. Khan, A. Sayyed, H. S. Al-Raweshidy, Zone Routing Protocol: How does it perform the other way round?, in Proc International Conference on ICT Convergence (ICTC), South Korea, 2012.

19. Iranmanesh, S, Raad, R, Kwan-Wu Chin A novel destination-based routing protocol (DBRP) in DTNs, in Proc International Symposium on Communications and Information Technologies (ISCIT), Gold Coast, Australia,2012.

20. Ghassan Samara, Munir Al-okour, Optimal Number of Cluster Heads in Wireless Sensors Networks Based on LEACH International Journal of Advanced Trends in Computer Science and Engineering, pp. 891 - 895, February 2020 https://doi.org/10.30534/ijatcse/2020/127912020 